\title{
Weekly Low-Dose Methotrexate Therapy for Sarcoidosis
}

\author{
Takafumi Suda, Atsuhiko Sato, Mikio Toyoshima, Shiro Imokawa, Atsushi Yoshitomi, Ryoji Tamura, \\ Hideki Suganuma, Takeshi Yagi, Hiroshi Hayakawa, Masahiro Shirai and Kingo Chida
}

\begin{abstract}
Low-dose methotrexate therapy has been used to treat a variety of chronic inflammatory diseases; a few studies have discussed the efficacy of this therapy for sarcoidosis. We present a patient with symptomatic sarcoidosis who was successfully treated with low-dose methotrexate after being refractory to steroids. This patient showed significant clinical improvement without development of any severe side effects. These findings suggest that low-dose methotrexate therapy may be a useful alternative treatment for sarcoidosis.

(Internal Medicine 33: 437-440, 1994)
\end{abstract}

Key words: refractory sarcoidosis, steroid-sparing effect

\section{Introduction}

Methotrexate is a folic acid antagonist that is used as an antimetabolite at high doses to treat malignancy. Recently, lowdose methotrexate therapy has been shown to be effective in the treatment of a variety of chronic inflammatory diseases, such as psoriasis, inflammatory bowel disease, rheumatoid arthritis, and bronchial asthma (1-4). However, there have been few reports on the efficacy of methotrexate for sarcoidosis (5-8). We report herein a patient with steroid-resistant sarcoidosis who was successfully treated with low-dose methotrexate.

\section{Case Report}

A 39-year-old man was admitted to our hospital for the evaluation of chest $X$-ray findings in 1984 . The chest X-ray film showed bilateral hilar lymphadenopathy and small nodular infiltrates in the upper lung fields. A diagnosis of sarcoidosis was made on the basis of uveitis, elevated serum angiotensin converting enzyme (SACE) level, and the detection of sarcoid granulomas by transbronchial lung biopsy (TBLB). The lung specimens revealed scattered, well-demarcated, non-caseating epithelioid granulomas with little interstitial fibrosis, involving predominantly the interstitium. Four years later, chest X-ray films showed marked shrinkage of upper lobes due to pulmonary fibrosis and he developed a productive cough. Since 1987, he received $60 \mathrm{mg}$ of prednisolone on alternate days, but the respiratory symptoms progressively worsened and his pulmonary function deteriorated.

In July 1991, he was readmitted for further treatment. On physical examination, breath sounds were diminished at the bilateral lung apices, but no rales were audible. The heart sounds were normal with no murmurs. Nodular skin lesions with a keloid-like appearance were found on both sides of the knees.

Laboratory findings showed a white blood cell count of $4,500 / \mathrm{mm}^{3}$, an erythrocyte sedimentation rate (ESR) of $45 \mathrm{~mm} /$ $\mathrm{h}$, a serum lactic dehydrogenase (LDH) level of $450 \mathrm{WU}$ (normal range: $100-400 \mathrm{WU}$ ), and a serum IgG concentration of $2,155 \mathrm{mg} / \mathrm{dl}(1,300-1,800 \mathrm{mg} / \mathrm{dl})$. The SACE level was 15 $\mathrm{IU} / \mathrm{l}(8.3-21.4 \mathrm{IU} / \mathrm{l})$, and the serum lysosome level was slightly elevated to $14.2 \mu \mathrm{g} / \mathrm{ml}(5.0-10.2 \mu \mathrm{g} / \mathrm{ml})$. There were no abnormalities on the ECG and Holter ECG recordings. The tuberculin skin test revealed a negative reaction. Biopsy of the nodular skin lesions proved them to be non-caseating epithelioid granulomas.

The chest X-ray film demonstrated consolidations and nodular infiltrates which predominantly affected the upper lung fields (Fig. 1A), and high resolution computed tomography (HRCT) showed conglomated masses and nodules with distortion of the lung parenchyma (Fig. 2A). An increased uptake of ${ }^{67}$ Ga-citrate in the lungs was seen (Fig. 3A). Pulmonary function tests revealed a mild restrictive impairment $(\mathrm{FVC}, 2.63 \mathrm{~L}$; \%FVC, 66.2; FEV1, 2.20 L), and arterial blood gas analysis showed a pH of 7.411, a $\mathrm{PCO}_{2}$ of 37.2 Torr, and a $\mathrm{PO}_{2}$ of 78.8 Torr.

In September 1991, he was started on oral methotrexate at $7.5 \mathrm{mg}$ once a week. Clinical improvement was noted after 23 weeks. His productive cough gradually subsided and there was a significant improvement in pulmonary function, as assessed by the FVC and arterial blood gases (Fig. 4). Addition-

From the Second Division, Department of Internal Medicine, Hamamatsu University School of Medicine, Hamamatsu

Received for publication December 20, 1993; Accepted for publication April 25, 1994

Reprint requests should be addressed to Dr. Takafumi Suda, the Second Division, Department of Internal Medicine, Hamamatsu University School of Medicine, 3600 Handa, Hamamatsu, 431-31 

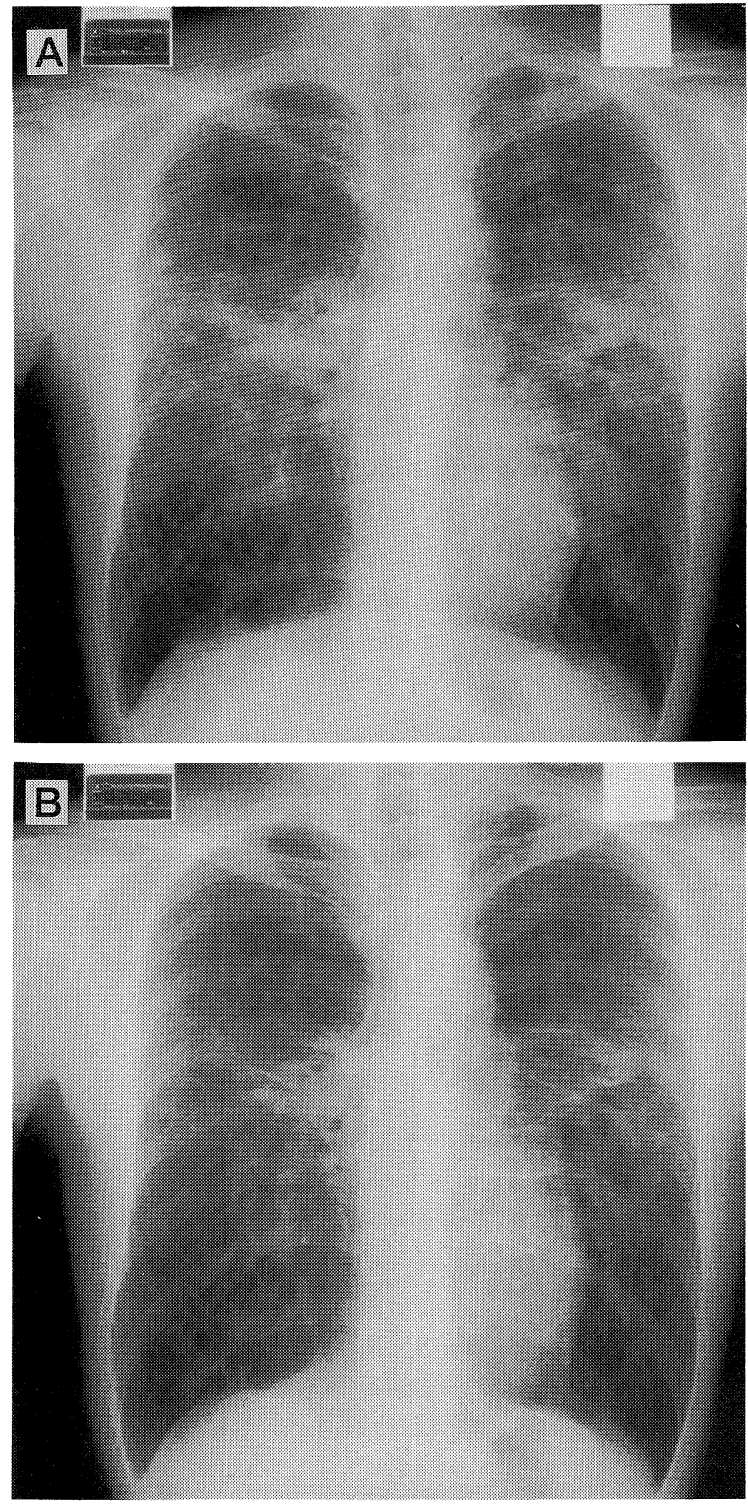

Fig. 1. Chest X-ray films. (A) On admission. Note consolidations and nodular infiltrates predominantly in upper lung fields. (B) After 3 months of therapy. Note the marked decrease in consolidations and nodular infiltrates.

ally, there was a decrease of the ESR, serum LDH level, and SACE level. The chest X-ray findings were also markedly improved (Fig. 1B), and the nodules and alveolar consolidation detected by HRCT showed a decrease (Fig. 2B). Furthermore, the lung uptake of Ga-citrate was significantly diminished (Fig. 3B). After treatment, the percentage and number of BAL lymphocytes decreased (Table 1), and the number of CD4+ T lymphocytes and HLA-DR+ lymphocytes also decreased. However, the CD4:CD8 ratio of BAL T lymphocytes showed a marked increase. Even the skin lesions improved slightly. Although he experienced nausea during the first week of therapy, this was resolved in $2-3$ weeks without specific treat-
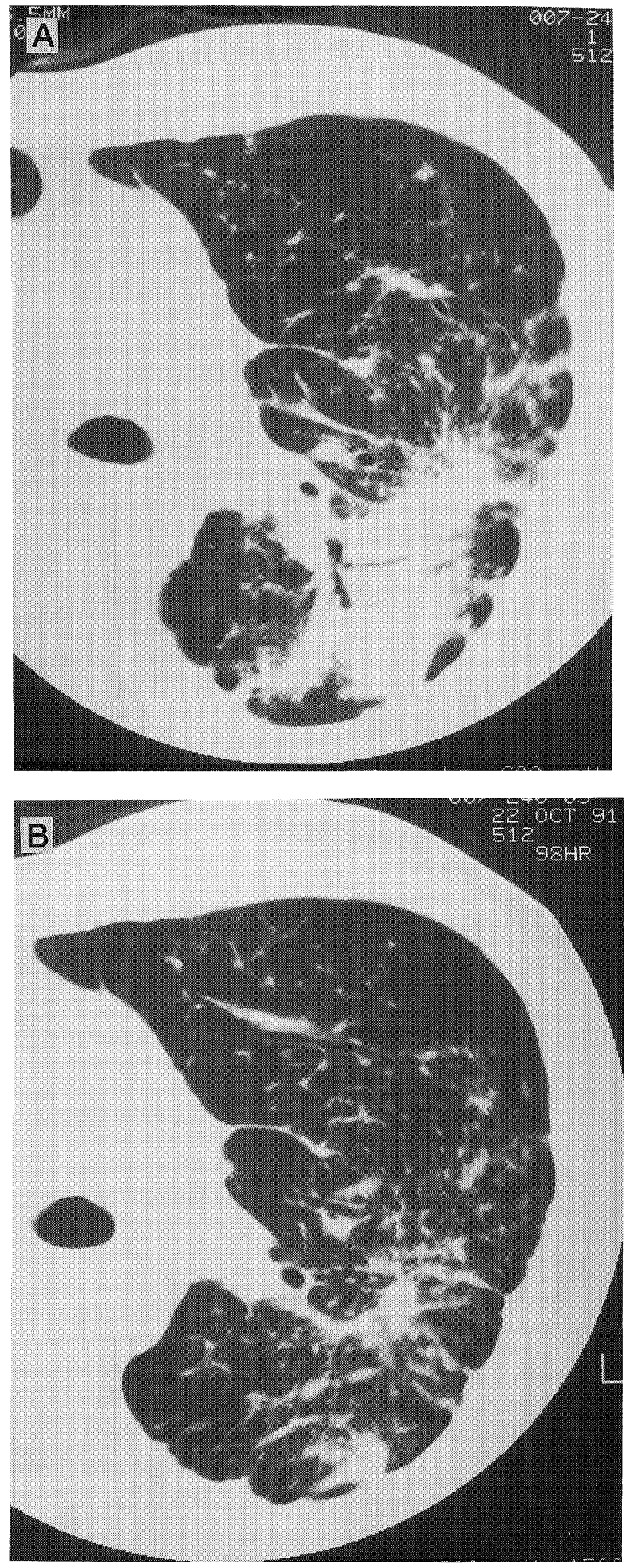

Fig. 2. High resolution computed tomography (HRCT) scans of chest. (A) On admission. Note conglomated mass and nodules. (B) After 3 months of therapy, the opacities showed a significant decrease. 

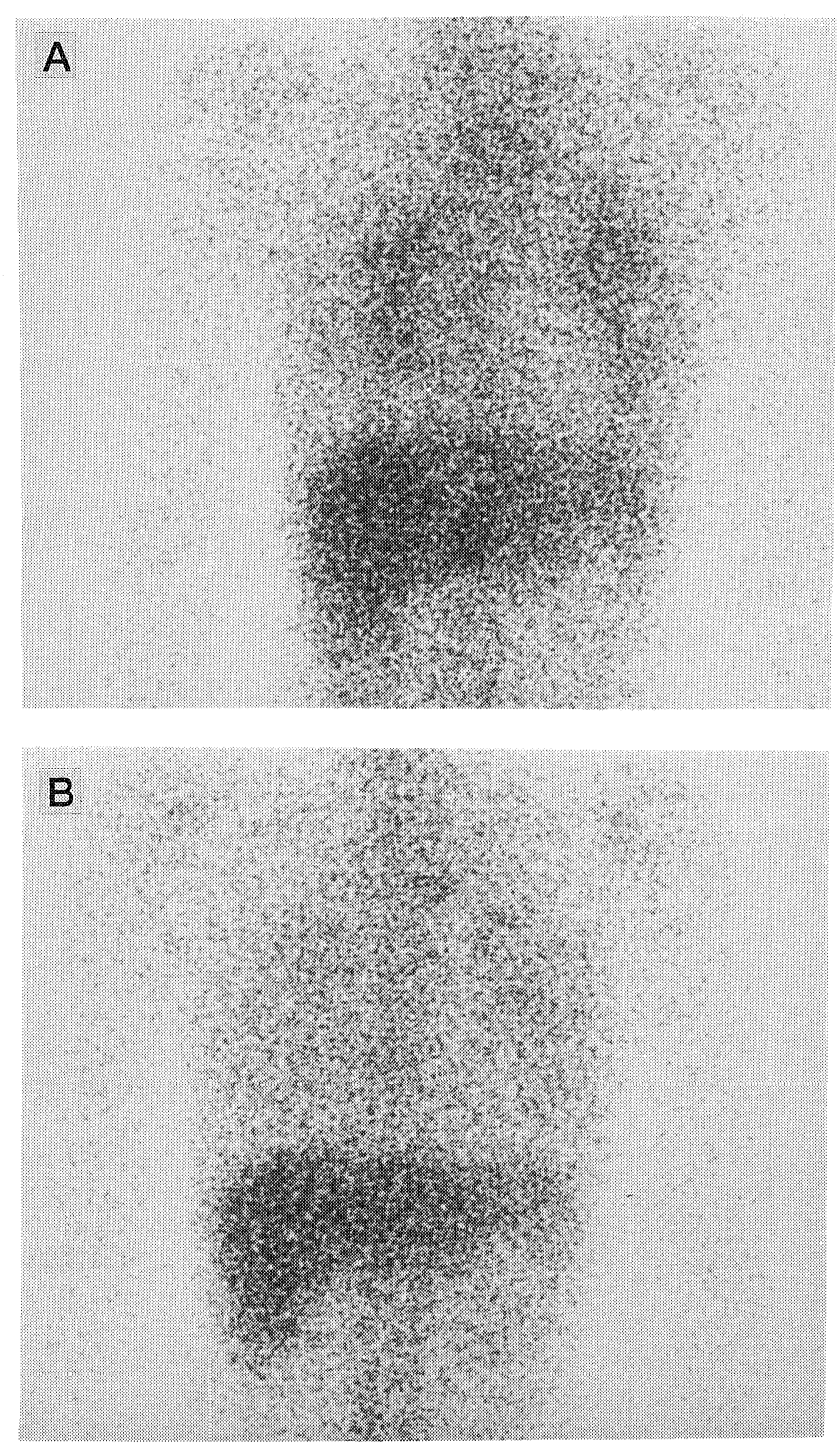

Fig. 3. $\quad{ }^{67}$ Ga-citrate scintigraphy. (A) On admission. Note the increased uptake in the lung. (B) After 3 months of therapy, the lung uptake was markedly diminished.

ment. No severe side effects, including hematologic or hepatic toxicity, were observed. After 6 months of therapy, the methotrexate regimen was changed to administration every 2 weeks. However, his condition remained stable and no deterioration occurred until 6 months later.

\section{Discussion}

Low-dose methotrexate therapy was first used as a treatment for psoriasis (1) and thereafter was used for several other inflammatory diseases. For example, recent studies have demonstrated the efficacy of low-dose methotrexate for rheumatoid arthritis and bronchial asthma $(3,4)$. This drug has been found to have a steroid-sparing effect and to cause no serious adverse reactions at low doses.
Despite the increasing use of low-dose methotrexate therapy for a variety of inflammatory diseases, only a few reports have suggested the efficacy of this therapy for sarcoidosis (5-8). Israel reported that patients with progressive sarcoidosis who show steroid resistance or steroid intolerance respond to methotrexate at a dose of $20 \mathrm{mg}$ twice weekly (5). It was also shown that administration of methotrexate at $25 \mathrm{mg}$ weekly led to a significant improvement in cutaneous sarcoidosis (6). More recently, Lower demonstrated that the administration of methotrexate at $10 \mathrm{mg}$ weekly is effective in chronic symptomatic sarcoidosis without severe side effects, and has a steroidsparing effect (8).

In the present sarcoidosis patient, a significant improvement was seen with weekly low-dose methotrexate treatments. Although his disease was resistant to steroid therapy, it responded well to methotrexate and there were no severe side effects. Clinical improvement was observed within 1 month. Methotrexate is known to cause dose-related adverse side effects, such as pancytopenia, liver damage, and pneumonitis. However, low-dose methotrexate only rarely causes hematologic, hepatic, or pulmonary toxicity (9-11), and the most common problem is gastrointestinal symptoms. Although the present patient complained of nausea during the initial phase of therapy, he soon became tolerant of the drug. Thus, our findings confirm that low-dose methotrexate is useful alternative therapy for patients with sarcoidosis who show resistance or intolerance for steroids.

The mechanism by which low-dose methotrexate acts in sarcoidosis remains unclear. In rheumatoid arthritis, several studies have demonstrated that low-dose methotrexate has an antiinflammatory effect, without an immunosuppressive effect on humoral or cell-mediated immunity (12). Low-dose methotrexate has also been shown to decrease neutrophil chemotaxis in response to C5a and LB4 $(13,14)$. In addition, it has been found that this therapy decreases hydrogen peroxide and TNF release by alveolar macrophages as well as reducing the number of BAL lymphocytes and the CD4:CD8 ratio of BAL T lymphocytes in sarcoidosis (15). These findings suggest that low-dose methotrexate has a direct effect on macrophages and $\mathrm{T}$ lymphocytes at the sites of inflammation.

The present patient treated with low-dose methotrexate also showed a decrease of BAL lymphocytes and CD4+ T lymphocytes after 3 months. However, CD8+ T lymphocytes also decreased markedly, resulting in an increase in the CD4:CD8 ratio. The reason for this increase in the $\mathrm{CD} 4: \mathrm{CD} 8$ ratio of $\mathrm{BAL}$ $\mathrm{T}$ lymphocytes despite significant clinical improvement, is unclear. Although the CD4:CD8 ratio is generally considered to reflect disease activity in sarcoidosis, some patients have been found to improve despite an increase of this ratio in BAL T lymphocytes, therefore it may not necessarily be correlated with disease activity.

The present findings indicated that low-dose methotrexate therapy is effective for the treatment of sarcoidosis and has low toxicity. However, it is unclear whether or not this therapy can improve the long-term prognosis of sarcoidosis by preventing pulmonary fibrosis and impairment of lung function. Thus, 


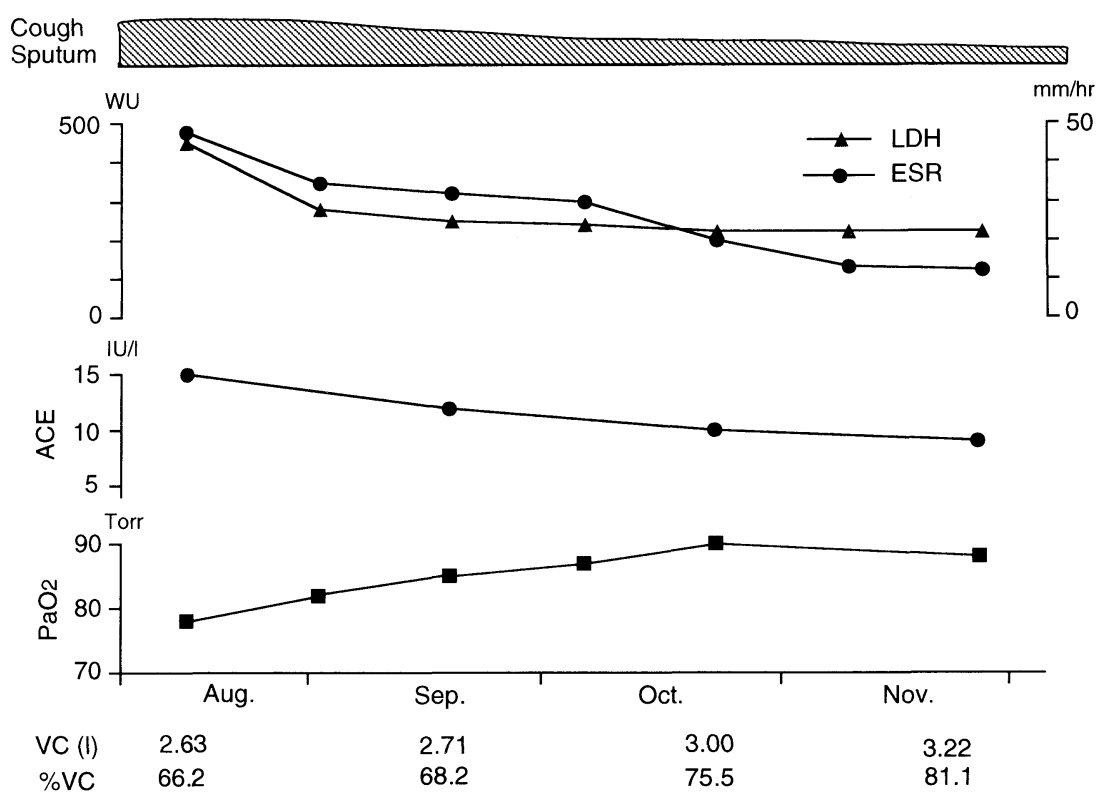

Fig. 4. Clinical course.

Table 1. Effects of Low-Dose Methotrexate Therapy on BALF Findings

\begin{tabular}{lcc}
\hline & Before therapy & 3 months of therapy \\
\hline Recovery rate, \% & 55.5 & 56.5 \\
Total cell count, $10^{4} / \mathrm{ml}$ BALF & 7.66 & 7.96 \\
& & \\
Differential cell count, $\%$ & & \\
$\left(10^{4} /\right.$ ml BALF $)$ & & \\
Macrophages & $77.6(5.94)$ & $86.3(6.87)$ \\
Lymphocytes & $17.5(1.34)$ & $11.7(0.93)$ \\
Neutrophils & $0.3(0.02)$ & $1.5(0.12)$ \\
Eosinophils & $4.6(0.35)$ & $0.5(0.04)$ \\
& & \\
Phenotypes of lymphocytes, \% & & \\
$\left(10^{3} /\right.$ ml BALF $)$ & & $69.4(6.45)$ \\
CD3 & $73.3(9.82)$ & $65.6(6.10)$ \\
CD4 & $51.8(6.94)$ & $13.4(1.25)$ \\
CD8 & $25.3(3.39)$ & $58.7(5.46)$ \\
HLA-DR & $67.8(9.08)$ & 4.90 \\
CD4/CD8 & 2.05 & \\
\hline
\end{tabular}

further follow-up studies are needed to assess the long-term efficacy and safety of low-dose methotrexate therapy for sarcoidosis.

\section{References}

1) Roenigk HH Jr, Fowler-Bergfeld W, Curtis GH. Methotrexate for psoriasis in weekly oral doses. Arch Dermatol 99: 86, 1969.

2) Kozarek RA, Patterson DJ, Gelfand MD, Botoman VA, Ball TJ, Wilske
KR. Methotrexate induces clinical and histological remission in patients with refractory inflammatory bowel disease. Ann Intern Med 110: 353, 1989.

3) Kremer JM, Lee JK. A long-term prospective study of the use of methotrexate in rheumatoid arthritis. Arthritis Rheum 31: 577, 1988.

4) Mullarkey MF, Blumenstein BA, Andrade WP, et al. Methotrexate in the treatment of corticosteroid-dependent asthma. New Engl J Med 318: 603, 1988.

5) Israel HL. Treatment of sarcoidosis. Thorax 20: 57, 1971.

6) Veien NK, Brodthagen H. Cutaneous sarcoidosis treated with methotrexate. Br J Dermatol 97: 213, 1977.

7) Soriano FG, Caramelli P, Nitrini R, Rocha AS. Neurosarcoidosis: therapeutic success with methotrexate. Postgrad Med J 66: 142, 1990.

8) Lower EE, Baughman RP. The use of low dose methotrexate in refractory sarcoidosis. Am J Med 299: 153, 1990.

9) Shupack JL, Webster GF. Pancytopenia following low-dose oral inethotrexate therapy for psoriasis. JAMA 259: 3594, 1988.

10) Lanse SB, Arnold GL, Gowans JDC, Kaplan MM. Low incidence of hepatotoxicity associated with long-term, low-dose oral methotrexate in treatment of refractory psoriasis, psoriatic arthritis, and rheumatoid arthritis. Dig Dis Sci 30: 104, 1985.

11) Carson CW, Cannon GW, Egger MJ, Ward JR, Clegg DO. Pulmonary disease during the treatment of rheumatoid arthritis with low dose pulse methotrexate. Semin Arthritis Rheum 16: 186, 1987.

12) Weinblatt ME, Coblyn JS, Fox DA, et al. Efficacy of low-dose methotrexate in rheumatoid arthritis. N Engl J Med 312: 818, 1985.

13) Ternowitz T, Bjerring $\mathrm{P}$, Andersen $\mathrm{PH}$, et al. Methotrexate inhibits the human C5a-induced skin response in patients with psoriasis. J Invest Dermatol 89: 192, 1987.

14) Van De Kerkhof PCM, Bauer FW, Maasen DE, et al. Methotrexate inhibits the leukotriene $\mathrm{B}_{4}$ induced intraepidermal accumulation of polymorphonuclear leukocytes. Br J Dermatol 113: 251a, 1985.

15) Baughman RP, Lower EE. The effect of corticosteroid or methotrexate therapy on lung lymphocytes and macrophages in sarcoidosis. Am Rev Respir Dis 142: 1268, 1990. 